\title{
Valence and Core-Level X-ray Photoelectron Spectroscopy of a Liquid Ammonia Microjet
}

Tillmann Buttersack, ${ }^{\dagger, \mathbb{I}}$ Philip E. Mason, ${ }^{\dagger, \mathbb{I}}$ Ryan S. McMullen, ${ }^{\ddagger}, \mathbb{I}$ Tomas Martinek, ${ }^{\dagger}$ Krystof Brezina, ${ }^{\dagger}$ Dennis Hein, ${ }^{\|}$Hebatallah Ali, ${ }^{\perp}$ Claudia Kolbeck, ${ }^{\perp}$ Christian Schewe, ${ }^{\perp}$ Sebastian Malerz, ${ }^{\perp}$ Bernd Winter, ${ }^{*}, \perp_{\odot}$ Robert Seidel, ${ }^{\|}$Ondrej Marsalek, ${ }^{*} \S_{\odot}$ Pavel Jungwirth, ${ }^{*},{ }^{\dagger}$ and Stephen E. Bradforth* $*$;0

${ }^{\dagger}$ Institute of Organic Chemistry and Biochemistry, Czech Academy of Sciences, Flemingovo nam. 2, 16610 Prague 6, Czech Republic

${ }^{\ddagger}$ Department of Chemistry, University of Southern California, Los Angeles, California 90089-0482, United States

${ }^{\S}$ Faculty of Mathematics and Physics, Charles University, Ke Karlovu 3, 12116 Prague 2, Czech Republic

"Helmholtz-Zentrum Berlin für Materialien und Energie, D-14109 Berlin, Germany

${ }^{\perp}$ Fritz-Haber-Institut der Max-Planck-Gesellschaft, D-14195 Berlin, Germany

Supporting Information

ABSTRACT: Photoelectron spectroscopy of microjets expanded into vacuum allows access to orbital energies for solute or solvent molecules in the liquid phase. Microjets of water, acetonitrile and alcohols have previously been studied; however, it has been unclear whether jets of low temperature molecular solvents could be realized. Here we demonstrate a stable $20 \mu \mathrm{m}$ jet of liquid ammonia ( -60 ${ }^{\circ} \mathrm{C}$ ) in a vacuum, which we use to record both valence and core-level band photoelectron spectra using soft X-ray synchrotron radiation. Significant shifts from isolated ammonia in the gas-phase are observed, as is the liquidphase photoelectron angular anisotropy. Comparisons with spectra of ammonia in clusters and the solid phase, as well as spectra for water in various phases potentially reveal how hydrogen bonding is reflected in the condensed phase electronic structure.

T iquid ammonia is often used as a medium for organic 1 chemistry due its ability to support long-lived highly reducing solvated electrons, first demonstrated by Birch in 1944. ${ }^{1}$ Solvated electrons can be generated simply by adding an alkali metal to anhydrous liquid ammonia; the solvated electrons have lifetimes on the order of months for solutions which are free from oxygen and moisture. It can be expected that photoelectron (PE) spectroscopy of these liquid solutions may reveal insights as to the binding energies of different electron motifs in ammonia. ${ }^{2,3}$ However, in a recent review of the spectroscopy of excess electrons in ammonia ${ }^{4}$ it is remarked that the electronic structure of neat liquid ammonia itself has not as yet been characterized through its photoelectron spectrum, although the PE spectrum of large ammonia clusters $^{5}$ and ammonia ice ${ }^{6}$ on $\mathrm{MoS}_{2}$ has been reported. The liquid PE spectrum would be a crucial complement to the known ammonia orbital energies in both vapor ${ }^{7}$ and amorphous ice; ${ }^{6}$ it is certainly a necessary prerequisite for an understanding of the band gap in liquid ammonia ${ }^{8}$ and to provide a more rounded understanding of excess electrons ${ }^{9}$ in liquid ammonia. A further interest in the liquid ammonia photoelectron spectrum is the comparison of the two simplest hydrogen bonded liquids: the question of how electronic structure evolves from isolated molecular $\mathrm{H}_{2} \mathrm{O}$ or $\mathrm{NH}_{3}$ through molecular clusters all the way to (bulk) liquid, and the effect of hydrogen bond strength on the orbital binding energies. Shifts from the gas phase in each band in the PE spectrum of liquid water are well-known not to be uniform reflecting different solvent-induced changes to individual molecular orbitals. ${ }^{10}$

A prerequisite to progress on the electronic structure of $\mathrm{NH}_{3(\mathrm{aq})}$ is to demonstrate the generation of a stable liquid jet of ammonia to enable photoelectron studies in vacuum. This paper provides proof-of-principle photoelectron spectroscopy from a liquid jet of $\mathrm{NH}_{3}$. The technique of forcing a room temperature liquid jet into vacuum was first used to obtain photoelectron spectra for liquid water, simple alcohols and nonane in $1997,{ }^{11}$ with the first photoelectron spectrum of water using synchrotron extreme ultraviolet recorded in 2004. ${ }^{10}$

Liquid jets of cryogenic nitrogen and argon were achieved by Faubel in 2001 using a simple metal aperture, ${ }^{12}$ with a system cooled all the way to the outlet aperture. Liquid ammonia poses a different challenge to these liquids. It is a gas until cooled below $-33{ }^{\circ} \mathrm{C}$ at atmospheric pressure; it was difficult for us to predict whether the liquid ammonia will immediately become a gas once a jet filament is introduced into vacuum due to ammonia's high vapor pressure $(10 \mathrm{kPa})$ even at -70 ${ }^{\circ} \mathrm{C}$. Also, it was not clear that if a jet is formed, the length of the laminar flow region would be sufficient to collect high signal-to-noise photoelectron spectra.

A liquid ammonia sampling system, submerged in a cold ethanol bath held at $-60{ }^{\circ} \mathrm{C}$, was employed. A $50 \mathrm{mM} \mathrm{KBr}$ solution was used to diminish the streaming potential, in analogy with water liquid jets. ${ }^{13}$ High-purity argon was

Received: October 10, 2018

Published: January 23, 2019 
introduced into the head space of the sample cylinder to force the liquid ammonia through $6 \mathrm{~mm}$ dimeter steel tubing to a 20 $\mu \mathrm{m}$ diameter nozzle by pressurization ( $5 \mathrm{bar}$ ), keeping the line at the same temperature until reaching the beginning of the glass jet nozzle.

PE experiments were carried out with the SOL ${ }^{3} \mathrm{PES}$ experimental setup ${ }^{14}$ at the variable polarization UE52-SGM1 beamline at the synchrotron radiation facility BESSY II. $^{15}$ Valence PE spectra were recorded using a photon energy of $310 \mathrm{eV}$ and core PE spectra were recorded with a photon energy of $640 \mathrm{eV}$; polarization with respect to the lab frame was varied relative to the facility floor. The energy resolution of the UE52-SGM1 beamline was better than $90 \mathrm{meV}$ for $310 \mathrm{eV}$ photon energy and $260 \mathrm{meV}$ for $640 \mathrm{eV}$, respectively. The electron analyzer offered a resolution of $125 \mathrm{meV}$ at the pass energy of $100 \mathrm{eV}$ used in our experiments. The rectangular Xray spot size was $60 \mu \mathrm{m} \times 100 \mu \mathrm{m}$ (exit slit) with the liquid ammonia microjet $\sim 20 \mathrm{~cm}$ behind the focus, such that the spot size at the liquid jet was roughly $400 \mu \mathrm{m}$.

A liquid jet of ammonia is formed with an argon backing pressure of 5 bar. After priming, a laminar flow region is formed 1-5 cm long; a stable liquid jet can be maintained for hours with a base chamber pressure of $\sim 4 \times 10^{-3} \mathrm{mbar}$ until the exhaustion of the reservoir (flow rate of about $0.5 \mathrm{~mL} / \mathrm{min}$; jet velocity $\sim 30 \mathrm{~m} / \mathrm{s}$ ). Unlike with water, evaporative cooling does not lead to ice formation as part of the flow.

The recorded photoelectron spectra are shown in Figure 1. Calibration of the absolute binding energies in the photoelectron spectrum of liquid ammonia was carried out by measuring the lowest binding energy $3 \mathrm{a}_{1} \mathrm{PE}$ band at consecutively lowered photon fluxes until both gas and liquid phase peaks no longer shifted due to the charging of the jet caused by ionized ammonia molecules (see Figure S1). Then the valence spectrum is calibrated against the literature gasphase lowest vertical ionization potential of $10.93 \mathrm{eV}{ }^{7}$ We find a gas-liquid shift of $1.84 \mathrm{eV}$, giving the liquid $3 \mathrm{a}_{1}$ peak at $9.1 \pm$ $0.1 \mathrm{eV}$. The calibrated valence photoelectron (Figure 1, top) spectrum shows, as expected, three bands for the gas and three bands that can be assigned to the liquid. The large gas-phase contribution is due to the high vapor pressure around the liquid ammonia jet as well as the large X-ray illuminated volume.

By subtracting the appropriately normalized gas phase spectrum, the PE peaks due to the liquid can be obtained (Figure 1 middle, Figure S2) and fit by three Gaussians (Figure S2, red line). A discernible shift to lower binding energies for all features arising from the liquid-phase is evident (see Table 1). A similar lowering of binding energies of the valence orbitals is also seen for the valence peaks in liquid water, but the shifts here are greater than for water. ${ }^{10}$ Furthermore, as for water, the shifts for the different bands are not the same. In ammonia, the shift is smallest for the lone pair $3 a_{1}$, which is the orbital affected most by $\mathrm{H}$-bond donation. A subtlety here is that the 1e band is split already in the gas phase due to degeneracy breaking in the resultant ${ }^{2} \mathrm{E}$ cation state (see Supporting Information). The binding energies for the $2 \mathrm{~s}$ dominated orbitals (labeled $2 \mathrm{a}_{1}$ in both water and ammonia) are shifted the most in both liquids.

The liquid PE spectrum resembles that observed for large ammonia clusters. ${ }^{5}$ Compared to amorphous ammonia ice, the $\mathrm{PE}$ features are shifted to a greater extent from the gas phase. The solid phase $3 \mathrm{a}_{1}$ band peak at $9.5 \mathrm{eV}$ is almost $2 \mathrm{eV}$ wide and is distinctly asymmetric unlike the liquid phase peak,

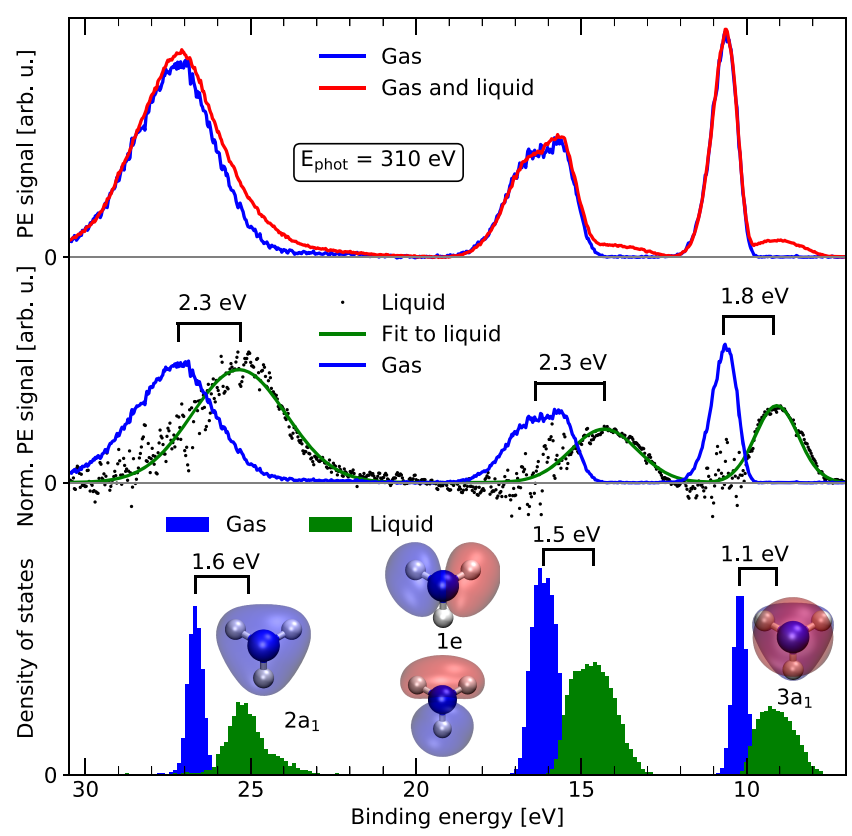

Figure 1. (Top) Valence band photoelectron spectra of $\mathrm{NH}_{3}$ (combined gas contribution and liquid given in red line) and the gas phase $\mathrm{NH}_{3}$ spectrum only (blue line) both measured at $310 \mathrm{eV}$ photon energy. In both spectra a Shirley-like background has been subtracted. Spectra are calibrated using the $3 \mathrm{a}_{1}$ position of liquid $\mathrm{NH}_{3}$. Assignments are made to parent isolated molecular orbitals (shown) by closeness in binding energy to gas-phase bands. All binding energies are with respect to the vacuum level. Polarization of the radiation is parallel to the electron collection direction. (Middle) Subtraction of the intensity-normalized experimental spectra reveals the liquid-only contribution (black dots, green fit) which areanormalized is compared to photoelectron bands for gas-phase (blue) ammonia. (Bottom) Same comparison now with theoretical PE bands calculated using the $\mathrm{G}_{0} \mathrm{~W}_{0}$ method. Fitting results are summarized in Table 1; residuals to fit are shown in the Supporting Information.

which is shifted to $9.1 \mathrm{eV}$ and more or less Gaussian. The 1e band in ammonia ice peaks at $15.1 \mathrm{eV}$ (and is also $\sim 2 \mathrm{eV}$ broad shading to the low BE side), whereas in liquid we see a further decrease in $\mathrm{BE}$ to $14.3 \mathrm{eV}$ for the band peak.

The angular anisotropy of the valence PE spectrum has been recorded but for a different photon energy, $265 \mathrm{eV}$ (Figure S3). For the $3 a_{1}$ and 1e bands, which are well separated from their vapor counterparts, the electron angular distributions are characterized by $\beta\left(3 \mathrm{a}_{1}\right)=1.5 / 1.0$ (gas/liquid) and $\beta(1 \mathrm{e})=$ $1.3 / 0.9$ (gas/liquid), respectively.

We have modeled the valence photoelectron bands using hybrid density functional calculations employing the $\mathrm{G}_{0} \mathrm{~W}_{0}$ approach. Calculations for the gas phase spectrum originate from a set of 5000 single point $\mathrm{G}_{0} \mathrm{~W}_{0}$ calculations for geometries sampled every 10 fs from a 50 ps long ab initio molecular dynamics (AIMD) trajectory of a single ammonia molecule. For liquid phase spectra, we simulated 64 ammonia molecules in a box employing periodic boundary conditions; for 100 configurational snapshots separated by 2 ps from a 200 ps run, the 256 highest $G_{0} W_{0}$ energies (i.e., four energies per $\mathrm{NH}_{3}$ molecule) were used for obtaining the quasi-particle energy distribution to model the spectra. Full details of the computational methodology are given in the Supporting Information.

The results of our calculations are summarized in the bottom part of Figure 1. First, we reproduced well the gas 
Table 1. Binding Energies and Full Width at Half Maxima (FWHM) of All Molecular Orbitals ${ }^{a}$

\begin{tabular}{|c|c|c|c|c|}
\hline orbital & $1 \mathrm{a}_{1}(\mathrm{~N} 1 \mathrm{~s})$ & $2 \mathrm{a}_{1}$ & 1e & $3 a_{1}$ \\
\hline BE liquid $(e V)$ & $403.49 \pm 0.05$ & $25.36 \pm 0.02$ & $14.31 \pm 0.03$ & $9.09 \pm 0.05$ \\
\hline fwhm liquid $(\mathrm{eV})$ & $1.3 \pm 0.1$ & $3.35 \pm 0.04$ & $2.38 \pm 0.08$ & $1.54 \pm 0.04$ \\
\hline lit. BE gas (eV) & $405.5(2)^{b}$ & $27.7^{d}$ & $16.6^{c}$ & $10.93^{c}$ \\
\hline calc. BE liquid $(\mathrm{eV})^{e}$ & - & 25.07 & 14.63 & 9.09 \\
\hline calc. BE gas (eV) & - & 26.64 & 16.47 & 10.67 \\
\hline
\end{tabular}

${ }^{a}$ Error bars reflect fitting uncertainty. ${ }^{b}$ Ref $5 .{ }^{c}$ Ref $7 .{ }^{d}$ Ref $16 .{ }^{e}$ Aligned with experiment for the $3 a_{1}$ orbital.

phase valence ionization energies using the $\mathrm{G}_{0} \mathrm{~W}_{0}$ approach in terms of absolute peak positions. Small residual deviations are primarily due to approximations used in describing the electronic structure. Second, in the liquid phase, the absolute values of electronic orbital energies are not directly accessible due to the use of periodic boundary conditions. ${ }^{17-19}$ Therefore, the whole spectrum was shifted such that the first peak was aligned with the experiment at $-9.09 \mathrm{eV}$. After this alignment, the calculated liquid phase spectrum exhibits good agreement with experimental data in terms of peak positions but is systematically underestimating the gas-to-liquid shifts. Note that the somewhat larger widths of the experimental peaks compared to the calculated ones is likely due to combination of a neglect of nuclear quantum effects in the simulations and the possible contribution from inelastic scattering for the experiment liquid spectrum distorting peaks to the high binding energy side; the extent of inelastic scattering has so far only been determined for much slower electrons liberated in laser based spectrometers. ${ }^{20}$

As we expect the ammonia core $\mathrm{N}$ 1s levels to be sensitive to local environment in highly alkali-metal doped ammonia solutions, the $\mathrm{N} \mathrm{1s}\left(1 \mathrm{a}_{1}\right)$ photoelectron band for the neat liquid was recorded with a photon energy of $640 \mathrm{eV}$, and the result is presented in Figure 2. As before, a substantial gas phase peak is observed. Note that we calibrated the $\mathrm{N}$ 1s liquid peak, which is at $403.49 \mathrm{eV}$, by using the position of the valence $3 a_{1}$ liquid peak and not against the $N$ 1s gas phase peak, because we have not yet excluded charging effects due to high photon flux affecting the gas-liquid shift. This binding energy for the $\mathrm{N} 1 \mathrm{~s}$ is very similar in clusters with $\sim 1600$

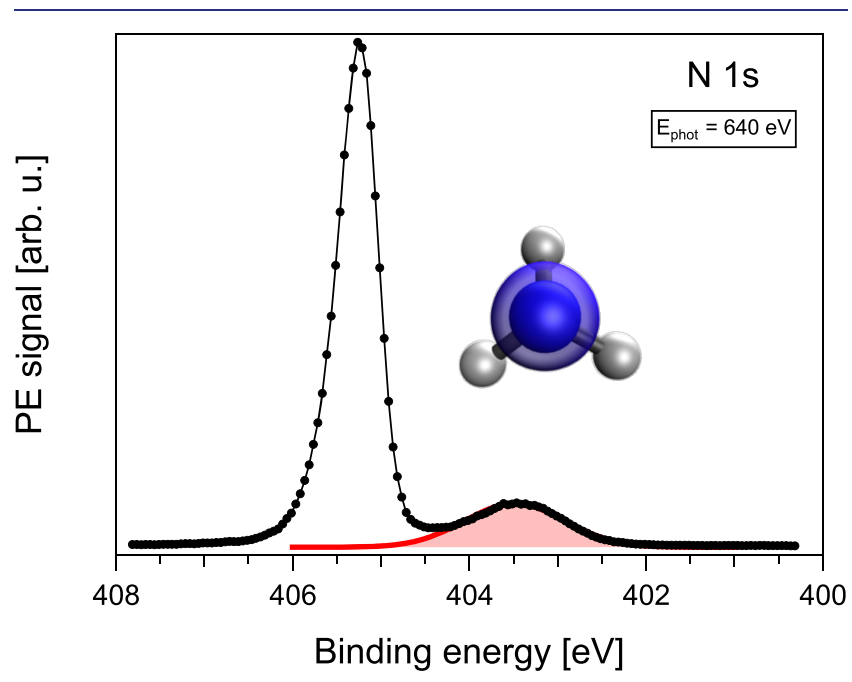

Figure 2. $1 \mathrm{a}_{1}(\mathrm{~N} \mathrm{1s})$ photoelectron spectrum of $\mathrm{NH}_{3}$ at $640 \mathrm{eV}$. The spectrum is calibrated using the position of the valence $3 a_{1}$ liquid peak measured under the same experimental conditions. Isolated molecule $\mathrm{N}$ 1s orbital shown. ammonia molecules suggesting that charging is not a significant problem. ${ }^{5}$ Using a literature value for the gas, ${ }^{5}$ the gas-liquid shift for this band is $2.0 \mathrm{eV}$, compared to a smaller shift of 1.77 $\mathrm{eV}$ on the $\mathrm{O} 1 \mathrm{~s}$ for water. The difference in the gas-liquid shifts between water and ammonia for the $\mathrm{N} 1 \mathrm{~s}$ and $\mathrm{O}$ 1s may in part reflect the different optical dielectric response of the two liquids via the Born stabilization of the final state. ${ }^{10}$

Finally, varying the X-ray polarization over six angles with respect to the detection direction for $\mathrm{N} 1 \mathrm{~s}$ PE band at $640 \mathrm{eV}$ (Figures S4 and S5) gives $\beta$ (gas/liquid) $=1.9 / 1.7$ showing only a small diminution of the photoelectron anisotropy due to elastic scattering of the outgoing electron through the liquid. ${ }^{21}$

In conclusion, we have presented the first measurements from a liquid ammonia microjet and demonstrated significant shifts in the photoelectron binding energies for both the valence and core orbitals of ammonia on condensation. The decrease in binding energies is more significant than those seen for amorphous ammonia ice deposited on $\mathrm{MoS}_{2}$. However, as for PE spectra from large clusters, we can clearly identify bands that match those from the isolated molecule. The basic shifts observed in experiment are recovered by calculations based on $\mathrm{ab}$ initio molecular dynamics and $\mathrm{G}_{0} \mathrm{~W}_{0}$ with a hybrid functional. Evidence for the degree of delocalization of the valence orbitals can be analyzed from the PE band anisotropy and peak splitting. Experiments that probe liquid ammonia increasingly doped with excess electrons are currently underway; the issue of delocalization and anisotropy will be particularly topical in this context and will be further explored in a future publication.

\section{ASSOCIATED CONTENT}

\section{S Supporting Information}

The Supporting Information is available free of charge on the ACS Publications website at DOI: 10.1021/jacs.8b10942.

Photon flux, angular distribution experimental data, as well as computational methodology and benchmarking (PDF)

\section{AUTHOR INFORMATION}

\section{Corresponding Authors}

*ondrej.marsalek@mff.cuni.cz

*winter@fhi-berlin.mpg.de

*pavel.jungwirth@uochb.cas.cz

*stephen.bradforth@usc.edu

ORCID

Bernd Winter: 0000-0002-5597-8888

Ondrej Marsalek: 0000-0002-8624-8837

Pavel Jungwirth: 0000-0002-6892-3288

Stephen E. Bradforth: 0000-0002-6164-3347

\section{Author Contributions}

II These authors contributed equally. 


\section{Notes}

The authors declare no competing financial interest.

\section{ACKNOWLEDGMENTS}

The authors thank the HZB staff prior to and during the beamtime for their assistance at BESSY II. P.E.M. acknowledges support from the viewers of his YouTube science channel. S.E.B. and R.S.M. are supported by the U.S. National Science Foundation (CHE-1665532). R.S.M. received additional funds from the States of Jersey Postgraduate Bursary. R.S. and B.W. gratefully acknowledge financial support from the German Research Foundation (DFG) within the Collaborative Research Center (SFB) 1109. R.S. also acknowledges an Emmy Noether Young Investigator stipend through the DFG (project SE 2253/3-1). O.M. was supported by the grant Primus16/SCI/27/247019. T.B. was supported by the Czech Ministry of Education and the EU (Grant CZ.02.2.69/ 0.0/0.0/16 027/0008477). P.J. acknowledges support from the Czech $\bar{S}$ cience Foundation (Grant P208/12/G016). The computational work was supported by the Czech Ministry of Education, Youth and Sports from the Large Infrastructures for Research, Experimental Development and Innovations project "IT4Innovations National Supercomputing Center LM2015070”.

\section{REFERENCES}

(1) Birch, A. J. 117. Reduction by Dissolving Metals. Part I. J. Chem. Soc. 1944, 430-437.

(2) Aulich, H.; Baron, B.; Delahay, P.; Lugo, R. Photoelectron Emission by Solvated Electrons in Liquid-Ammonia. J. Chem. Phys. 1973, 58 (10), 4439-4443.

(3) Hartweg, S.; West, A. H. C.; Yoder, B. L.; Signorell, R. Metal Transition in Sodium-Ammonia Nanodroplets. Angew. Chem. 2016, 128 (40), 12535-12538.

(4) Vöhringer, P. Ultrafast Dynamics of Electrons in Ammonia. Annu. Rev. Phys. Chem. 2015, 66 (1), 97-118.

(5) Lindblad, A.; Bergersen, H.; Pokapanich, W.; Tchaplyguine, M.; Öhrwall, G.; Bjorneholm, O. Charge Delocalization Dynamics of Ammonia in Different Hydrogen Bonding Environments: Free Clusters and in Liquid Water Solution. Phys. Chem. Chem. Phys. 2009, 11 (11), 1758-7.

(6) Yu, K. Y.; McMenamin, J. C.; Spicer, W. E. UPS Measurements of Molecular Energy Level of Condensed Gases. Surf. Sci. 1975, 50 (1), 149-156.

(7) Edvardsson, D.; Baltzer, P.; Karlsson, L.; Wannberg, B.; Holland, D. M. P.; Shaw, D. A.; Rennie, E. E. A Photoabsorption, Photodissociation and Photoelectron Spectroscopy Study of $\mathrm{NH}$ 3and ND 3. J. Phys. B: At., Mol. Opt. Phys. 1999, 32 (11), 2583-2609.

(8) Vogler, T.; Vöhringer, P. Probing the Band Gap of Liquid Ammonia with Femtosecond Multiphoton Ionization Spectroscopy. Phys. Chem. Chem. Phys. 2018, 20 (40), 25657-25665.

(9) Zurek, E.; Edwards, P. P.; Hoffmann, R. A Molecular Perspective on Lithium-Ammonia Solutions. Angew. Chem., Int. Ed. 2009, 48 (44), $8198-8232$.

(10) Winter, B.; Weber, R.; Widdra, W.; Dittmar, M.; Faubel, M.; Hertel, I. V. Full Valence Band Photoemission from Liquid Water Using EUV Synchrotron Radiation. J. Phys. Chem. A 2004, 108 (14), $2625-2632$.

(11) Faubel, M.; Steiner, B.; Toennies, J. P. Photoelectron Spectroscopy of Liquid Water, Some Alcohols, and Pure Nonane in Free Micro Jets. J. Chem. Phys. 1997, 106 (22), 9013-9031.

(12) Wieland, M.; Wilhein, T.; Faubel, M.; Ellert, C.; Schmidt, M.; Sublemontier, O. EUV and Fast Ion Emission from Cryogenic Liquid Jet Target. Appl. Phys. B: Lasers Opt. 2001, 72 (5), 591-597.

(13) Kurahashi, N.; Karashima, S.; Tang, Y.; Horio, T.; Abulimiti, B.; Suzuki, Y.-I.; Ogi, Y.; Oura, M.; Suzuki, T. Photoelectron Spectros- copy of Aqueous Solutions: Streaming Potentials of $\mathrm{NaX}(\mathrm{X}=\mathrm{Cl}, \mathrm{Br}$ and I) Solutions and Electron Binding Energies of Liquid Water and X-. J. Chem. Phys. 2014, 140 (17), 174506.

(14) Seidel, R.; Pohl, M. N.; Ali, H.; Winter, B.; Aziz, E. F. Advances in Liquid Phase Soft-X-Ray Photoemission Spectroscopy: a New Experimental Setup at BESSY II. Rev. Sci. Instrum. 2017, 88 (7), 073107.

(15) Helmholtz Zentrum Berlin für Materialien und Energie, The Variable Polarization Undulator Beamline UE52 SGM at BESSY II. J. Large-Scale Res. Facilities 2016, 2, A70.

(16) Banna, M. S.; Shirley, D. A. Molecular Photoelectron Spectroscopy at $132.3 \mathrm{eV}$. the Second-Row Hydrides. J. Chem. Phys. 1975, 63 (11), 4759-4766.

(17) Chen, W.; Ambrosio, F.; Miceli, G.; Pasquarello, A. Ab Initio Electronic Structure of Liquid Water. Phys. Rev. Lett. 2016, 117 (18), 186401.

(18) Ambrosio, F.; Guo, Z.; Pasquarello, A. Absolute Energy Levels of Liquid Water. J. Phys. Chem. Lett. 2018, 9 (12), 3212-3216.

(19) Gaiduk, A. P.; Pham, T. A.; Govoni, M.; Paesani, F.; Galli, G. Electron Affinity of Liquid Water. Nat. Commun. 2018, 9 (1), 247.

(20) Luckhaus, D.; Yamamoto, Y.-I.; Suzuki, T.; Signorell, R. Genuine Binding Energy of the Hydrated Electron. Sci. Adv. 2017, 3 (4), e1603224.

(21) Thürmer, S.; Seidel, R.; Faubel, M.; Eberhardt, W.; Hemminger, J. C.; Bradforth, S. E.; Winter, B. Photoelectron Angular Distributions from Liquid Water: Effects of Electron Scattering. Phys. Rev. Lett. 2013, 111 (17), 173005. 